\title{
The Role of Cognitive Reserve in Recovery from Traumatic Brain Injury
}

\author{
Kayla A. Steward, BS ${ }^{1}$, Richard Kennedy, MD, $\mathrm{PhD}^{2}$, Thomas A. Novack, $\mathrm{PhD}^{3}$, Michael \\ Crowe, $\mathrm{PhD}^{1}$, Daniel C. Marson, JD, $\mathrm{PhD}^{4}$, and Kristen L. Triebel, PsyD ${ }^{4}$ \\ ${ }^{1}$ Department of Psychology, University of Alabama at Birmingham, Birmingham, AL, USA \\ ${ }^{2}$ Department of Medicine, University of Alabama at Birmingham, Birmingham, AL, USA \\ ${ }^{3}$ Department of Physical Medicine and Rehabilitation, University of Alabama at Birmingham, \\ Birmingham, AL, USA \\ ${ }^{4}$ Department of Neurology, University of Alabama at Birmingham, Birmingham, AL, USA
}

\begin{abstract}
Objective-To examine whether cognitive reserve (CR) attenuates the initial impact of TBI on cognitive performance (neural reserve) and results in faster cognitive recovery rates in the first year post-injury (neural compensation), and whether the advantage of CR differs based on the severity of TBI.
\end{abstract}

Setting-Inpatient/outpatient clinics at academic medical center.

Participants-Adults with mild TBI (mTBI; $n=28)$, complicated mild TBI (cmTBI; $n=24)$, and moderate-to-severe TBI (msevTBI; $n=57)$, and demographically-matched controls $(n=66)$.

Design-Retrospective; longitudinal cohort assessed at 1-, 6-, and 12-months post-injury.

Main Measures-Outcomes were 3 cognitive domains: processing speed/executive function, verbal fluency, and memory. Premorbid IQ, estimated with the Wechsler Test of Adult Reading, served as CR proxy.

Results-Higher premorbid IQ was associated with better performance on cognitive domains at one-month post-injury, and the effect of IQ was similarly beneficial for all groups. Cognitive recovery rate was moderated only by TBI severity; those with more severe TBI had faster recovery in the first year.

Conclusion-Results support only the neural reserve theory of CR within a TBI population and indicate that CR is neuroprotective regardless of the degree of TBI. Higher premorbid CR does not allow for more rapid adaptation and recovery from injury.

\section{Keywords}

traumatic brain injury; cognitive reserve; neuropsychology; rehabilitation; longitudinal studies

Corresponding Author: Kayla A. Steward, B.S., Department of Psychology, University of Alabama at Birmingham, Campbell Hall 415, $15303^{\text {rd }}$ Avenue South, Birmingham, Alabama 35294-1170. Phone: (832) 891-3162. ksteward@uab.edu.

Conflicts of Interest: The authors declare no conflicts of interest. 
Traumatic brain injury (TBI) affects 1.5 to 2 million Americans each year ${ }^{1,2}$ and is often associated with deficits in processing speed, attention, memory, language, visuospatial processing, and executive functioning. ${ }^{3,4}$ While some individuals experience a quick cognitive recovery and return to pre-morbid functioning within 3 months after TBI, others maintain deficits a decade or longer despite rehabilitation attempts. ${ }^{4}$ Researchers have noted that injury severity, as measured by post-traumatic amnesia (PTA), loss of consciousness (LOC), and/or Glasgow Coma Scale (GCS), is a major predictor of immediate and long-term cognitive outcome following $\mathrm{TBI}^{3}$; however, there is significant variability in cognitive outcomes even between individuals classified with the same TBI severity. ${ }^{5,6}$ Given the high personal, medical, and societal costs of rehabilitation services ${ }^{7}$, it is not feasible to prescribe extensive treatment programs to everyone. Therefore, it is crucial to find additional variables that can reliably predict which patients would benefit most from an aggressive treatment approach during the rehabilitative process. In addition, being able to reliably predict cognitive outcome and rate of recovery would assist clinicians in advising patients and families on issues such as returning to work, driving, and living independently.

Recently, studies have begun to apply the theory of cognitive reserve (CR) to TBI populations in order to explain this variability in cognitive outcomes following TBI (see Mathias \& Wheaton [2015] for review ${ }^{8}$ ). Broadly, CR refers to the cognitive networks used when completing mental tasks. It was initially hypothesized to be a protective factor in the face of age- or disease-related neurodegeneration..$^{9-11}$ As CR is a theoretical concept, it is often measured using proxies, such as estimated premorbid intelligence and/or length of exposure to cognitively stimulating life events (i.e. educational attainment, challenging occupations, mentally stimulating leisure activities). The theory of CR can be divided into "neural reserve" and "neural compensation" models, which are not necessarily exclusive ${ }^{9-11}$ :

The neural reserve model states that there are individual differences in the efficiency and capacity of a person's cognitive processing systems, which protect these networks from disruption following injury to the brain. Essentially, when neuropathological damage is equivalent between individuals, those with higher CR will demonstrate fewer cognitive and functional deficits relative to those with lower CR. ${ }^{9-11}$ Numerous studies have determined that higher premorbid educational and occupational attainment is associated with better immediate and long-term post-injury cognitive and functional outcome, ${ }^{12-19}$ providing support for this theory. Interestingly, two studies have found evidence that the effect of CR may differ depending on the severity of TBI. Jeon et al. (2008) ${ }^{17}$ found that having higher CR was a protective factor for adults with TBI, but the effect weakened as TBI severity increased. Similarly, Fay et al. $(2010)^{20}$ determined that having lower CR was a risk factor for worse outcome in children with complicated mild TBI (cmTBI), but not for those with mild TBI (mTBI). The results of these studies indicate that individuals may reach a level of neuronal and axonal injury at which pre-injury $\mathrm{CR}$ is no longer beneficial to their recovery.

The neural compensation model of CR posits that certain individual's existing brain networks are more capable of adaptation and network reorganization following injury. This model posits that individuals with higher $\mathrm{CR}$ are able to recover more rapidly from 
pathological disruption of preexisting networks by enlisting compensatory networks (i.e., neuroplasticity). ${ }^{9-11}$ Longitudinal studies assessing whether CR predicts rate of cognitive recovery are critical for providing evidence for the compensation model in a TBI population. Unfortunately, few studies have approached this topic using statistical methods that allow for prediction of rate of change (e.g., mixed modeling). These studies failed to find a relationship between $\mathrm{CR}$ and recovery trajectories in the first year post-injury on any of the cognitive or functional domains assessed. ${ }^{21-24}$ As previously stated, some evidence exists that the benefit of CR during recovery may be tempered in those with more severe head injury. ${ }^{17,20}$ Since all of these studies primarily involved individuals with moderate-to-severe TBI (msevTBI), it is possible that the compensation model applies only to those with milder TBI; however, this has yet to be investigated.

To our knowledge, this is the first study to assess whether CR, as measured by estimated pre-morbid IQ, predicts cognitive outcome at one month post-injury and rate of recovery over the first year post-injury using the full spectrum of TBI severity (healthy controls, mTBI, cmTBI, and msevTBI). First, we hypothesized that those with higher levels of CR would have better initial post-injury cognitive function (neural reserve). Second, we hypothesized that those with higher levels of CR would have faster cognitive recovery rates in the first year after injury (neural compensation). Third, we examined whether the benefit of CR differs based on TBI severity. We hypothesized that relative to controls, the advantage of CR on initial cognitive outcome will weaken as TBI severity level increases. We also hypothesized that, relative to controls, CR will have the strongest effect on rate of recovery for those with msevTBI in comparison to the other TBI groups.

\section{METHODS}

\section{Participants}

Participants with TBI ( $n=109)$ and demographically-matched controls $(n=66)$ were enrolled from the University of Alabama at Birmingham (UAB) between 2007 and 2011 as part of a larger NIH-funded longitudinal study investigating medical decision making in TBI. ${ }^{25}$ Consistent with the protocol established by the TBI Model System program, participants with TBI were recruited from the UAB hospital system and initially assessed within a window of 2 to 6 weeks after injury, with a goal of assessing at one month after injury. ${ }^{26}$ Participants were also assessed at 6- and 12-months post-injury with a 2-week scheduling window on either side.

A board-certified rehabilitation neuropsychologist assigned a TBI severity level of mTBI $(n=28)$, cmTBI $(n=24)$, or msevTBI $(n=57)$ using diagnostic criteria which has been welldescribed previously ${ }^{27-29}$ (see Table 1). Individuals with penetrating brain injuries (e.g., gunshot wound) were excluded from the study. Patients were excluded if they had received substance abuse treatment within 1 year of enrollment (per patient/family report) or had a preexisting diagnosed central nervous system disorder, developmental disorder, or severe psychiatric disorder. Individuals with a prior mTBI were included if their previous injury occurred at least 1 year before enrollment; however, all those with a history of msevTBI were excluded. 
Healthy controls were recruited through local advertisements and selected to match participants with TBI on demographic variables of age, gender, ethnicity, and education. Controls were excluded if they had been diagnosed with a psychiatric disorder (except mild depression), substance abuse, cerebrovascular disease, or other neurologic disease. None of the controls were taking medications known to affect cognition.

Participants completed a battery of neuropsychological measures at each visit. Written informed consent was obtained from each participant or a legally authorized representative. The UAB Institutional Review Board approved the study procedures. All enrolled patients with TBI were capable of participating in the cognitive evaluation as judged by the study neuropsychologist, although not all patients had achieved full orientation at the time of the baseline assessment.

Twenty-five patients with TBI and 17 controls did not complete all three visits due to the following reasons: scheduling problems (7\%), no longer interested in participating (7\%), moved/distance (5\%), and unknown/other (81\%). Non-completers did not differ from completers with regards to demographic variables, estimated IQ, TBI classification/GCS score, or baseline cognitive outcomes.

\section{Measures}

Wechsler Test of Adult Reading-Word pronunciation tests are commonly used "hold" tests, which are neuropsychological measures thought to be unaffected by neurological damage. They have been used as premorbid IQ estimates in a variety of cognitively impaired populations. ${ }^{30-33}$ One such word pronunciation task is the Wechsler Test of Adult Reading (WTAR). ${ }^{34}$ The WTAR is comprised of 50 words with irregular pronunciations that participants read aloud. The raw score can be transformed to an age-adjusted standard score, which is used together with the participant's demographic information (age, gender, race, education) to predict IQ $(M=100 ; S D=15)$. The WTAR was co-normed with the Wechsler Adult Intelligence Scale, Third Edition (WAIS-III) ${ }^{35}$ and has been validated even in populations exhibiting questionable effort. ${ }^{36}$

Neuropsychological measures-All participants were administered a standardized battery of neuropsychological tests shown to be helpful in distinguishing TBI-related cognitive deficits from normal cognition. ${ }^{37}$ As a data reduction step to control the number of experiment-wise analyses, composite cognitive indices were created by transforming raw neuropsychological test scores into z-scores using published normative data, referenced below. Domains were created by averaging the z-scores for each test within the domain $(\mathrm{M}=0, \mathrm{SD}=1)$. The domains are as follows:

1. processing speed/executive function: Trail Making Test (TMT) Part A and $B,{ }^{38}$ Digit Symbol Coding and Symbol Search subtests from the WAIS-III ${ }^{35}$

2. verbal fluency: animal fluency, fruit/vegetable fluency, and clothing fluency ${ }^{39}$; and

3. memory: California Verbal Learning Test, second edition (CVLT-II) ${ }^{40}$ (Trials 15 total recall, short delay free recall, and long-delay free recall), Logical Memory 
I and II (immediate and delayed recall) subtest of the Wechsler Memory Scale, third edition (WMS-III). ${ }^{41}$

If a participant was missing over half of their individual raw test scores for a domain, they were excluded from analyses. Otherwise, we averaged the tests that they had available.

\section{Data analysis}

Demographic and clinical variables were compared between the four groups using one-way analysis of variance (ANOVA) for continuous variables and Pearson's chi-square tests for dichotomous variables.

Mixed effects models were used to examine the interaction between injury severity and CR on the initial status and rate of change over time (or slope). Separate models were used for each of the three cognitive domains. Mixed models were chosen for analyses as they incorporate all participants in the estimation, regardless of how many waves of data they contribute. ${ }^{42,43}$ Data was first modeled using an unconditional means model, which describes and partitions outcome variation in the absence of any within- or between-subject predictors. Next, data was modeled using Level 1 (i.e., "unconditional growth") models, which incorporated the effect of time (measured in months; centered on one-month postinjury, which was the initial time point of assessment). Because individuals only completed a maximum of three visits, a linear effect for time was selected. Level 1 models assessed whether there was sufficient variation in initial status and growth trajectories that other predictor variables might explain. These parameters (initial status, rate of change) are then treated as outcomes in Level 2 (i.e., "conditional growth") models. These models assess whether between-subjects variables, including WTAR-predicted IQ (centered on population mean of 100), TBI severity (controls as reference group) and the interaction between IQ $\mathrm{x}$ TBI severity, predict initial status and/or moderate rate of change (i.e., have a significant interaction with 'Time'). As both the outcome variables and WTAR-predicted IQ were already adjusted for demographic variables, these were not added as covariates to the model. Final Level 2 models were constructed using only significant predictors from the prior model. Restricted maximum likelihood was used for parameter estimation. An unstructured error covariance structure was selected, as it is the least restrictive and recommended for analyses using few waves of data. ${ }^{42}$ The Aikaike Information Criterion (AIC) was used to determine whether the inclusion of additional predictors improved the model over the previous one. The assigned significance level for analyses was $p<.05$.

\section{RESULTS}

\section{Demographic and sample characteristics}

Table 2 lists the causes of TBI. Table 3 presents demographic/clinical variables for the groups. Individuals with cmTBI were older than the controls and those with msevTBI, there was a higher proportion of non-white participants in the mTBI group compared to the other two TBI groups, and participants with msevTBI had a higher percentage of males than all other groups (all $\mathrm{p}<.05$ ). As expected, the msevTBI group had the lowest Galveston Orientation and Amnesia Test (GOAT) scores at one-month post-injury. Of note, the 
msevTBI group had significantly lower estimated IQ than controls (mean difference of 9.8 IQ points); however, they were still well within the normative "average" range.

\section{Cognitive outcome}

Table 4 presents the group means and standard deviations for each of the cognitive domains over time. Table 5 presents the estimated parameters from the two main fitted models (Level 2 conditional growth models with all hypothesized predictors and interactions and the final, reduced Level 2 models with only significant predictors) for each of the cognitive domains.

Examination of the variance components from the unconditional means model (not displayed in Table 5) revealed that there was sufficient between-subjects variation in processing speed/executive function, memory, and verbal fluency, accounting for $74.8 \%$, $61.0 \%$, and $62.5 \%$ of total variation for each domain, respectively. Variance components from Level 1 unconditional growth models (also not displayed in Table 5) suggested that the linear effect of time explained an adequate portion of within-person variation. In addition, there was significant between-person variation in initial status (but not in slope) remaining, suggesting that a Level 2 conditional growth model with between-subjects predictors would be beneficial. Finally, this model showed that higher initial cognitive status was negatively correlated with rate of change across all domains (all $\mathrm{p}<.05$ ).

The results from the initial Level 2 models, which included all hypothesized predictors and interactions, revealed that TBI severity and WTAR-estimated IQ, but not their interaction, significantly moderated initial level of impairment on all three cognitive domains. Only TBI severity significantly moderated rate of change for each of the cognitive domains.

The final Level 2 models included only these significant main effects. With regards to the effect of CR on outcome, a 10-point increase in estimated premorbid IQ was associated with a cognitive domain standard deviation increase of 0.31 for processing speed/executive function domain, 0.37 for memory, and 0.25 for verbal fluency (calculated by multiplying the effect of IQ by 10; all $\mathrm{p}<.001$ ).

At one-month post-injury, controls did not differ from the population mean on processing speed/executive function or memory domains; however, they did significantly improve over time in both of these domains, indicating practice effects on these measures. In contrast, controls performed approximately a quarter of a standard deviation lower than the population mean on verbal fluency at the first evaluation and remained stable over time. Individuals with mTBI performed significantly worse than controls at one-month post-injury on all domains, but their recovery trajectories did not differ from changes noted in performance among controls. Those with cmTBI and msevTBI performed worse than controls on all domains at one-month post-injury and had significantly more rapid rates of change than controls, reflecting recovery of cognitive abilities. Figure 1 displays average recovery on each cognitive domain by severity group and high/low predicted IQ.

Given that our TBI groups differed in premorbid IQ, models were re-analyzed with the effect of TBI severity excluded to determine whether TBI severity significantly accounted 
for the IQ effect. The effect of IQ remained nonsignificant, which indicates that the confounding of TBI severity and premorbid IQ did not markedly influence results.

\section{DISCUSSION}

This study found evidence that higher CR is associated with better cognitive performance in all tested domains, providing evidence for the neural reserve model of CR in this population. To illustrate the clinical significance of these findings, compare two individuals recovering from TBI: one with a high average premorbid IQ of 115 and the other with a low average IQ of 85. The individual with higher IQ performs 0.6 standard deviations better on the verbal fluency domain, 0.9 standard deviations better on the processing speed/executive function domain, and 1.2 standard deviations better on the memory domain shortly after injury in comparison to the individual with low average IQ. Given that this effect was identical for healthy, demographically matched controls and did not differ based on the degree of brain injury (i.e., there was no interaction between CR and TBI severity), these results did not support our hypothesis that having higher CR provides an additional buffer specifically for those with TBI. Importantly, we did not find evidence that CR reaches a level of damage at which it becomes less effective (i.e., evidence of a brain damage threshold). Rather, these results suggest that the positive correlation between intelligence and cognition that exists in healthy adults is preserved following TBI.

Contrary to our second hypothesis, we did not find that CR impacts rate of cognitive recovery following TBI. These results suggest that the compensation model, which states that $\mathrm{CR}$ enables more rapid restoration of function via reorganization of brain networks, cannot be extended to a population of individuals recovering from traumatic brain injury. One limitation of this work is that our first point of assessment was one month following injury, when some individuals, especially those with milder head injury, may have already experienced a full recovery. Future studies should seek to assess patients closer to the date of injury to capture the full recovery period and allow for more accurate prediction.

Additionally, cognitive trajectory was assessed at only three time points over the first year post-injury. Due to the relatively few time points, only linear slope could be estimated when in reality the recovery slope in likely asymptotic. ${ }^{44}$ It is possible that the imperfect fit of slope impacted our ability to find significance. Another possibility is that the effects on slope were too modest to detect with our relatively small sample size.

These results are consistent with Green et al.'s study ${ }^{21}$, which also found an association between estimated premorbid IQ and performance on simple processing speed, untimed executive function, and memory measures at two months post-injury in a msevTBI sample. Their study, along with others, also failed to find a moderating effect of CR variables on recovery trajectory. ${ }^{22-24}$

As expected, the severity of TBI negatively impacted initial performance and rate of recovery in all cognitive domains. Notably, we found differences in both initial status and rate of recovery between those with mild and complicated mild TBI. Clinicians should be aware that although an individual may fall in to the "mild TBI" category based on traditional diagnostic criteria (e.g., PTA, GCS, LOC), presence of intracranial damage (i.e., 
"complicated" mild TBI) will negatively impact prognosis. Due to the insufficient number of participants with moderate TBI, we were unable to separate them from the severe TBI group for analyses.

Another strength of the current study is that we included a wide breadth of cognitive domains in order to assess a larger spectrum of outcome in comparison to previous literature. Interestingly, across all groups, performance in the verbal fluency domain was the overall most impaired initially and had the slowest rate of improvement. Although statistical comparison of inter-domain differences is outside the scope of the current study, these results indicate that verbal fluency, which incorporates aspects of expressive language, processing speed, and executive function, may be particularly sensitive to the neuropathological effects of head injury. Targeting these skills heavily during the rehabilitation process may prove beneficial to outcome. Finally, it is important to note that individuals with an average level of intelligence fell within the "normal range" of functioning (+/- 0.25 SD) on the memory and processing speed/executive function domains by a year following TBI of any severity. Although there may still be some deviation in performance on individual tests within a domain, overall ability should be broadly intact in these domains for individuals with estimated premorbid IQ of 100 or more.

Interestingly, our msevTBI group's predicted IQ was nearly 10 points lower than the other groups despite similar demographics. There is evidence that word-reading tests may underestimate IQ in persons with moderate-to-severe $\mathrm{TBI}^{45-47}$, which could indicate that this measure does not accurately reflect the "cognitive reserve" concept in this subgroup. An ideal study would have information regarding intellectual performance before participants sustained their head injury, as this would be a true measure of premorbid IQ. However, this was not available for the current sample.

In conclusion, these results support the neural reserve theory of CR within a TBI population and there is no specific brain damage "threshold" at which reserve ceases to be beneficial. Our results suggest that the effect of CR is due to preservation of the relationship between intelligence and cognition following TBI. In contrast, having higher levels of premorbid CR does not allow for more rapid adaptation and recovery from injury. Thus, early intervention and cognitive rehabilitation efforts should be equally targeted regardless of estimated IQ. There are currently only a handful of studies using mixed modeling to determine which variables predict initial status and rate of recovery following injury. Additionally, this is the first of these studies to assess whether there is an interaction between cognitive reserve (i.e., IQ) and TBI severity on outcome and rate of change, using the full severity spectrum of TBI and matched controls. The results of this study and others in this area have concluded that many pre-injury variables do not appear to have a significant effect on recovery rate following TBI. These findings have favorable implications from a clinical perspective, since these are variables that are often difficult, if not impossible, to change. Therefore, future studies should turn to modifiable post-injury variables to account for variation in recovery. More detailed knowledge of the mechanisms influencing recovery will hopefully allow clinicians to further refine their ability in identifying survivors of TBI most at risk of cognitive impairment, individuals that are not recovering at expected rates, and develop 
treatment plans to address areas of cognitive function most likely to remain impaired following injury.

\section{Acknowledgments}

The authors thank the following contributors: Sandra Caldwell, MA (UAB Department of Physical Medicine and Rehabilitation, data collection); UAB Neuropsychology Laboratory Staff (data collection); Pat R. Pritchard, MD (UAB Department of Surgery, referring study participants) and Sarah Nafziger, MD (UAB Department of Emergency Medicine, referring study participants).

Source of Funding: This work was supported by the National Institute on Child Health and Human Development (grant no. 1R01HD053074) (Dr. Marson, PI).

\section{References}

1. NIH consensus development panel on rehabilitation of persons with traumatic brain injury. JAMA. 1999; 282:974-983. [PubMed: 10485684]

2. Rutland-Brown W, Langlois JA, Thomas K, Xi Y. Incidence of traumatic brain injury in the United States. J Head Trauma Rehabil. 2006; 21:544-548. [PubMed: 17122685]

3. Dikmen S, Corrigan JD, Levin HS, Machamer J, Stiers W, Weisskopf MG. Cognitive outcome following traumatic brain injury. J Head Trauma Rehabil. 2009; 24:430-438. [PubMed: 19940676]

4. Rabinowitz AR, Levin HS. Cognitive sequelae of traumatic brain injury. Psychiatr Clin North Am. 2014; 37:1-11. [PubMed: 24529420]

5. Lingsma HF, Roozenbeek B, Steyerberg EW, Murray GD, Maas AI. Early prognosis in traumatic brain injury: From prophecies to predictions. Lancet Neurol. 2010; 9:543-554. [PubMed: 20398861]

6. Millis SR, Rosenthal M, Novack TA, et al. Long-term neuropsychological outcome after traumatic brain injury. J Head Trauma Rehabil. 2001; 16:343-355. [PubMed: 11461657]

7. Faul M, Wald MM, Rutland-Brown W, Sullivent EE, Sattin RW. Using a cost-benefit analysis to estimate outcomes of a clinical treatment guideline: Testing the brain trauma foundation guidelines for the treatment of severe traumatic brain injury. J Trauma Inj Infect Crit Care. 2007; 63:12711278.

8. Mathias JL, Wheaton P. Contribution of brain or biological reserve and cognitive or neural reserve to outcome after TBI: A meta-analysis (prior to 2015). Neurosci Biobehav Rev. 2015; 55:573-593. [PubMed: 26054792]

9. Steffener J, Stern Y. Exploring the neural basis of cognitive reserve in aging. Biochim Biophys Acta. 2012; 1822:467-473. [PubMed: 21982946]

10. Stern Y, Habeck C, Moeller J, et al. Brain networks associated with cognitive reserve in healthy young and old adults. Cereb Cortex. 2005; 15:394-402. [PubMed: 15749983]

11. Stern Y. Cognitive reserve and Alzheimer disease. Alzheimer Dis Assoc Disord. 2006; 20:69-74.

12. Garcia-Molina A, Ensenat-Cantallops A, Sanchez-Carrion R, Rodriguez P, Tormos JM, RoigRovira T. Interindividual variability in recovery after traumatic brain injury: Effect of cognitive reserve. Med Clin (Barc). 2013; 140:527-531. [PubMed: 23481869]

13. Schneider E, Sur S, Raymont V, et al. Functional recovery after moderate/severe traumatic brain injury: A role for cognitive reserve? Neurology. 2014; 82:1636-1642. [PubMed: 24759845]

14. Sigurdardottir S, Andelic N, Roe C, Schanke A. Cognitive recovery and predictors of functional outcome 1 year after traumatic brain injury. J Int Neuropsychol Soc. 2009; 15:740-750. [PubMed: 19602303]

15. Novack T, Bush B, Meythaler J, Canupp K. Outcome after traumatic brain injury: Pathway analysis of contributions from premorbid, injury severity, and recovery variables. Arch Phys Med Rehabil. 2001; 82:300-305. [PubMed: 11245749]

16. Ponsford J, Draper K, Schonberger M. Functional outcome 10 years after traumatic brain injury: Its relationship with demographic, injury severity, cognitive and emotional status. J Int Neuropsychol Soc. 2008; 14:233-242. [PubMed: 18282321] 
17. Jeon I, Kim O, Kim M, Kim S, Chang C, Bai D. The effect of premorbid demographic factors on the recovery of neurocognitive function in traumatic brain injury patients. J Korean Neurosurg Soc. 2008; 44:295-302. [PubMed: 19119465]

18. Sumowski J, Chiaravalloti N, Krch D, Paxton J, DeLuca J. Education attenuates the negative impact of traumatic brain injury on cognitive status. Arch Phys Med Rehabil. 2013; 94:25622564. [PubMed: 23932968]

19. Kesler S, Adams H, Blasey C, Bigler E. Premorbid intellectual functioning, education, and brain size in traumatic brain injury: An investigation of the cognitive reserve hypothesis. Appl Neuropsychol. 2003; 10:153-162. [PubMed: 12890641]

20. Fay TB, Yeates KO, Taylor HG, et al. Cognitive reserve as a moderator of postconcussive symptoms in children with complicated and uncomplicated mild traumatic brain injury. J Int Neuropsychol Soc. 2010; 16:94-105. [PubMed: 19835663]

21. Green R, Colella B, Christensen B, et al. Examining moderators of cognitive recovery trajectories after moderate to severe traumatic brain injury. Arch Phys Med Rehabil. 2008; 89:S16-S24. [PubMed: 19081437]

22. Spitz G, Ponsford J, Rudzki D, Maller JJ. Association between cognitive performance and functional outcome following traumatic brain injury: A longitudinal multilevel examination. $\mathrm{J}$ Neuropsychol. 2012; 26:604-612.

23. Spikman JM, Timmerman ME, Zomeren van AH, Deelman BG. Recovery versus retest effects in attention after closed head injury. J Clin Exp Neuropsychol. 1999; 21:585-605. [PubMed: 10572280]

24. Chu BC, Mills S, Arango-Lasprilla JC, Hanks R, Novack T, Hart T. Measuring recovery in new learning and memory following traumatic brain injury: A mixed-effects modeling approach. J Clin Exp Neuropsychol. 2007; 29:617-625. [PubMed: 17691034]

25. Triebel KL, Martin RC, Novack TA, et al. Treatment consent capacity in patients with traumatic brain injury across a range of injury severity. Neurology. 2012; 78:1472-1478. [PubMed: 22496195]

26. Kalmar K, Novack TA, Nakase-Richardson R, et al. Feasibility of a brief neuropsychologic test battery during acute inpatient rehabilitation after traumatic brain injury. Arch Phys Med Rehabil. 2008; 89:942-949. [PubMed: 18452744]

27. Kashluba S, Hanks RA, Casey JE, Millis SR. Neuropsychologic and functional outcome after complicated mild traumatic brain injury. Arch Phys Med Rehabil. 2008; 89:904-911. [PubMed: 18452740]

28. Brasure, M., Lamberty, GJ., Sayer, NA., et al. Multidisciplinary postacute rehabiliation for moderate to severe traumatic brain injury in adults. Rockville, MD: Agency for Healthcare Research and Quality; 2012. (Prepared by the Minnesota Evidence-based Practice Center under Contract No. 290-2007-10064-I) AHRQ Comparative Effectiveness Reviews Publication No. 12EHC101-EF

29. Williams DH, Levin HS, Eisenberg HM. Mild head injury classification. Neurosurgery. 1990; 27:422-428. [PubMed: 2234336]

30. Dwan TM, Ownsworth T, Chambers S, Walker DG, Shum DH. Neuropsychological assessment of individuals with brain tumor: Comparison of approaches used in the classification of impairment. Front Oncol. 2015; 5:1-8. [PubMed: 25667919]

31. McGurn B, Starr JM, Topfer JA, et al. Pronunciation of irregular words is preserved in dementia, validating pre-morbid IQ estimation. Neurology. 2004; 62:1184-1186. [PubMed: 15079021]

32. Hanks RA, Millis SR, Ricker JH, et al. The predictive validity of a brief inpatient neuropsychologic battery for persons with traumatic brain injury. Arch Phys Med Rehabil. 2008; 89:950-957. [PubMed: 18452745]

33. Green RE, Melo B, Christensen B, Ngo LA, Monette G, Bradbury C. Measuring premorbid IQ in traumatic brain injury: An examination of the validity of the Wechsler Test of Adult Reading (WTAR). J Clin Exp Neuropsychol. 2008; 30:163-172. [PubMed: 18213530]

34. Wechsler, D. Wechsler Test of Adult Reading: WTAR. San Antonio, TX: The Psychological Corporation; 2001. 
35. Wechsler, D. WAIS-III Wechsler Adult Intelligence Scale-Third Edition. San Antonio, TX: The Psychological Corporation; 1997.

36. Whitney KA, Shepart PH, Mariner J, Mossbarger B, Herman S. Validity of the Wechsler Test of Adult Reading (WTAR): Effort considered in a clinical sample of U.S. military veterans. Appl Neuropsychol Adult. 2010; 17:196-204.

37. Lezak, M., Howieson, D., Loring, D. Neuropsychological Assessment. 4. New York: Oxford University Press; 2004.

38. Reitan, R., Wolfson, D. The Halstead-Reitan Neuropsychological Test Battery: Theory and Clinical Interpretation. Tuscon, AZ: Neuropsychology Press; 1993.

39. Spreen, O., Strauss, E. Compendium of Neuropsychological Tests: Administration, norms, and commentary. 2. New York, NY: Oxford University Press; 1998.

40. Delis, D., Kramer, J., Kaplan, E., Ober, B. CVLT-II California Verbal Learning Test. San Antonio, TX: The Psychological Corporation; 2000.

41. Wechsler, D. WMS-III Wechsler Memory Scale-Third Edition. San Antonio, TX: The Psychological Corporation; 1997.

42. Singer, JD., Willett, JB. Applied longitudinal data analysis: Modeling change and event occurrence. New York, NY: Oxford University Press; 2003.

43. Brown, H., Prescott, R. Applied mixed models in medicine. 2. Chichester: Wiley; 2006.

44. Christensen BK, Colella B, Inness E, et al. Recovery of cognitive function after traumatic brain injury: A multilevel modeling analysis of Canadian outcomes. Arch Phys Med Rehabil. 2008; 89:S3-S15.

45. Mathias JL, Bowden SC, Bigler ED, Rosenfeld JV. Is performance on the Wechsler Test of Adult Reading affected by traumatic brain injury? Br J Clin Psychol. 2007; 46:457-466. [PubMed: 17535531]

46. Orme DR, Johnstone B, Hanks R, Novack TA. The WRAT-3 reading subtest as a measure of premorbid intelligence among persons with brain injury. Rehabil Psychol. 2004; 49:250-253.

47. Steward KA, Novack TA, Kennedy R, Crowe M, Marson DC, Triebel KL. The Wechsler Test of Adult Reading as a measure of premorbid intelligence following traumatic brain injury. Arch Clin Neuropsychol. 2017; 32:98-103. [PubMed: 27799224] 


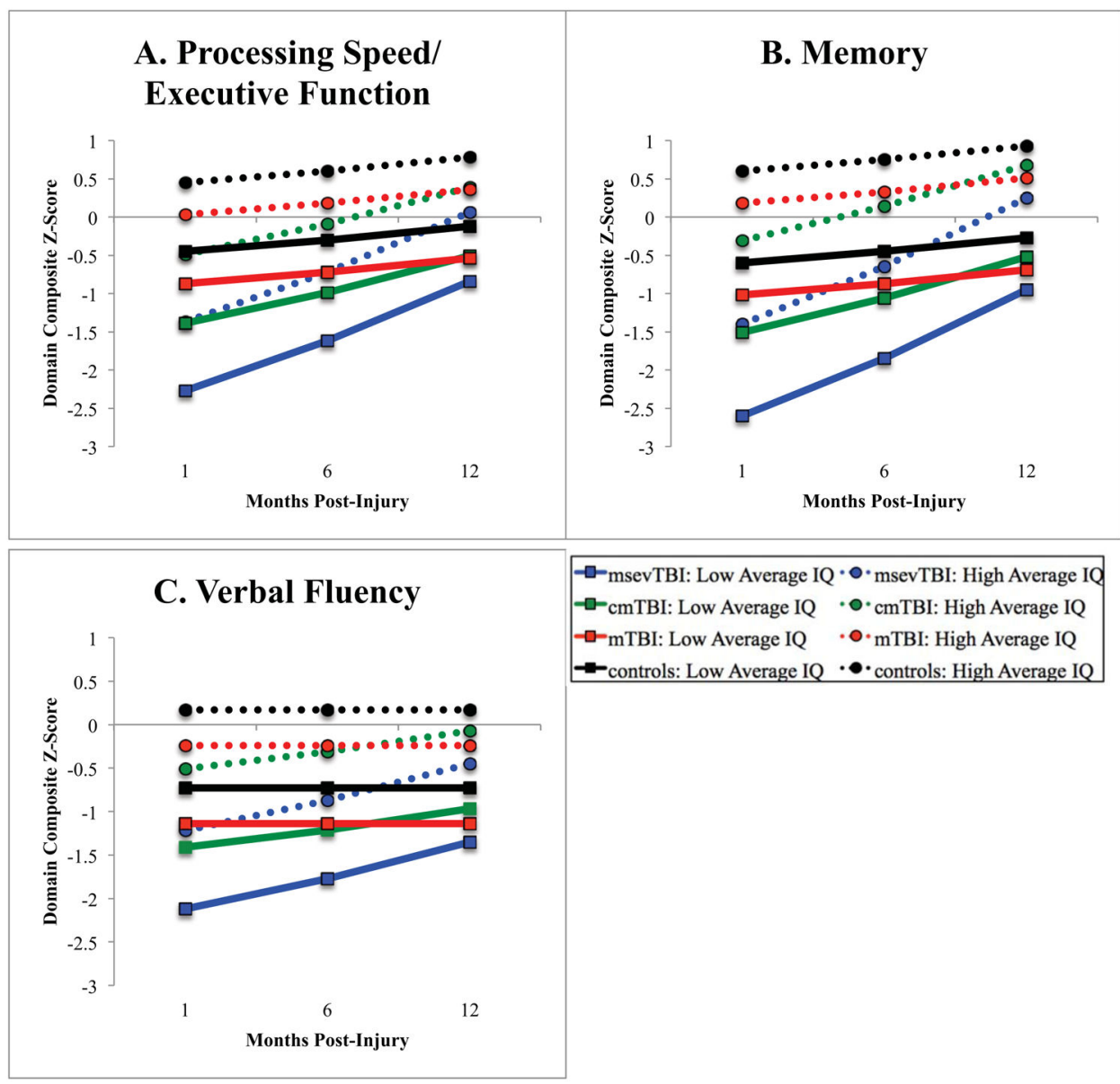

Figure 1.

Average cognitive recovery trajectories for each group split by estimated premorbid intelligence. Outcomes for each domain (Panels A, B, and C) are presented as Z-scores, which are standardized values with a population mean of 0 and standard deviation of 1 . WTAR-estimated IQ was dichotomized into high/low average categories (+/- 1 standard deviation from the population mean of 100) for the purposes of visualization only. 


\section{Table 1}

Criteria for TBI Severity Classification

\begin{tabular}{lclll}
\hline Severity & GCS & LOC & PTA & Structural Brain Changes* \\
\hline Mild TBI & $13-15$ & $\leq 30 \mathrm{~min}$. & $\leq 24 \mathrm{hrs}$. & No \\
Complicated Mild TBI & $13-15$ & $\leq 30 \mathrm{~min}$. & $\leq 24 \mathrm{hrs}$. & Yes \\
Moderate/Severe TBI & $\leq 12$ & $>30 \mathrm{~min}$. & $>24 \mathrm{hrs}$. & Yes \\
\hline
\end{tabular}

Abbreviation: GCS, Glasgow Coma Scale; LOC, Loss of consciousness; PTA, Post traumatic amnesia; TBI, Traumatic brain injury.

Note. Initial GCS scores were not available for intubated patients with msevTBI.

*

Cranial magnetic resonance imaging or computed tomography scans were used to determine evidence of structural brain changes, such as contusions, subdural hematoma, or diffuse axonal injury. 


\section{Table 2}

Causes of TBI in patient sample $(\mathrm{n}=109)$

\begin{tabular}{lcc}
\hline Cause & N & \% \\
\hline Car collision & 62 & 57 \\
Falls & 21 & 19 \\
Other motor vehicle collision (motorcycle, ATV, etc.) & 12 & 11 \\
Assault with blunt instrument & 5 & 5 \\
Bicycle & 3 & 3 \\
Sports & 2 & 2 \\
Other & 4 & 4 \\
\hline
\end{tabular}

Abbreviation: ATV, all-terrain vehicle. 
Table 3

Demographic and clinical characteristics of study participants

\begin{tabular}{lcccccc}
\hline Variables & Range & Controls $(\mathbf{n = 6 6})$ & mTBI $(\mathbf{n = 2 8})$ & $\mathbf{c m T B I}(\mathbf{n = 2 4})$ & $\mathbf{m s e v T B I}(\mathbf{n = 5 7})$ & $\boldsymbol{F} / \boldsymbol{\chi}^{\mathbf{2}}$ \\
\hline Age (y) & $19-79$ & $35.4 \pm 14.4$ & $35.4 \pm 12.4$ & $45.3 \pm 18.4$ & $35.5 \pm 14.8$ & $3.368^{a}$ \\
Gender (male) & & $40(60.6)$ & $15(53.6)$ & $13(54.2)$ & $45(78.9)$ & $8.220^{a}$ \\
Race (white) & & $48(72.7)$ & $15(53.6)$ & $20(83.3)$ & $48(84.2)$ & $10.465^{a}$ \\
Education (y) & $8-20$ & $12.9 \pm 1.5$ & $13.1 \pm 1.9$ & $13.9 \pm 2.8$ & $12.6 \pm 2.4$ & 2.232 \\
WTAR IQ & $68-125$ & $101.4 \pm 11.1$ & $97.1 \pm 11.6$ & $101.9 \pm 13.1$ & $91.6 \pm 14.5$ & $7.194 b$ \\
GOAT & $0-100$ & & & & & \\
1 mo & & N/A & $96.0 \pm 4.9$ & $95.3 \pm 6.8$ & $70.3 \pm 32.5$ & $14.928^{b}$ \\
6 mo & & N/A & $94.3 \pm 5.8$ & $96.6 \pm 6.0$ & $92.0 \pm 12.6$ & 1.615 \\
12 mo & & N/A & $93.1 \pm 6.4$ & $97.5 \pm 3.7$ & $94.4 \pm 15.2$ & 0.811 \\
GCS & 3-15 & N/A & $14.6 \pm 0.8$ & $14.7 \pm 0.6$ & $5.8 \pm 3.0$ & \\
\hline
\end{tabular}

NOTE. Values are mean \pm SD or n $(\%)$. Gender and race differences analyzed using Pearson chi-square. All other variables assessed using ANOVA. Differences in GCS were not assessed as this was used to classify TBI severity.

Abbreviations: GCS, Glasgow Coma Scale; GOAT, Galveston Orientation and Amnesia Test; N/A, Not Applicable; WTAR, Wechsler Test of Adult Reading; mTBI, mild TBI; cmTBI, complicated mild TBI; msevTBI, moderate to severe TBI.

${ }_{\mathrm{p}<.05}$,

$b_{\mathrm{p}<.001}$ 


\section{Table 4}

Means and standard deviations for the three cognitive domains at each time point across the TBI severity groups.

\begin{tabular}{lrrccc}
\hline Group & Months Post-injury & $\mathbf{N}$ & Processing Speed/Executive Function & Memory & Verbal Fluency \\
\hline Controls & 1 mo. & 66 & $0.14 \pm 0.69$ & $-0.16 \pm 0.95$ & $-0.23 \pm 0.75$ \\
& 6 mo. & 58 & $0.33 \pm 0.77$ & $0.22 \pm 1.07$ & $-0.20 \pm 0.75$ \\
& 12 mo. & 50 & $0.42 \pm 0.75$ & $0.43 \pm 0.94$ & $-0.10 \pm 0.77$ \\
Mild TBI & 1 mo. & 28 & $-0.51 \pm 0.94$ & $-0.58 \pm 0.91$ & $-0.81 \pm 0.90$ \\
& 6 mo. & 27 & $0.01 \pm 0.83$ & $-0.07 \pm 0.90$ & $-0.55 \pm 0.69$ \\
& 12 mo. & 26 & $-0.03 \pm 0.81$ & $-0.07 \pm 1.09$ & $-0.57 \pm 0.84$ \\
Complicated Mild TBI & 1 mo. & 24 & $-0.85 \pm 1.10$ & $-0.87 \pm 1.37$ & $-0.88 \pm 0.97$ \\
& 6 mo. & 21 & $-0.16 \pm 0.80$ & $-0.07 \pm 0.93$ & $-0.63 \pm 0.78$ \\
& 12 mo. & 17 & $-0.06 \pm 0.85$ & $-0.14 \pm 1.01$ & $-0.47 \pm 0.72$ \\
Moderate to Severe TBI & 1 mo. & 57 & $-2.13 \pm 0.80$ & $-2.46 \pm 1.05$ & $-2.01 \pm 0.58$ \\
& 6 mo. & 46 & $-0.96 \pm 0.78$ & $-1.01 \pm 1.14$ & $-1.25 \pm 0.72$ \\
& 12 mo. & 40 & $-0.65 \pm 0.89$ & $-0.96 \pm 1.16$ & $-1.10 \pm 0.60$ \\
\hline
\end{tabular}

NOTE. Values are averaged z-scores presented as mean \pm SD. Of those that completed the visit, the following numbers of subjects were excluded from specific domains because they were missing $>50 \%$ of the variables for the domain: At 1-month post-injury, 1 was excluded from Processing Speed/Executive Function, 7 were excluded from Verbal Fluency, and 2 were excluded from Memory. At 6-months post-injury, 1 was excluded from Memory. 


\section{Table 5}

Initial status and rate of change over 12-months for each of the cognitive domains $(n=175)$.

\begin{tabular}{|c|c|c|c|c|c|c|c|}
\hline & & \multicolumn{6}{|c|}{ Cognitive Domain } \\
\hline & & \multicolumn{2}{|c|}{ Processing Speed/Executive Function } & \multicolumn{2}{|c|}{ Memory } & \multicolumn{2}{|c|}{ Verbal Fluency } \\
\hline & & Full & Final & Full & Final & Full & Final \\
\hline \multirow[t]{8}{*}{ Initial Status } & Intercept & $.11(.09)$ & $.11(.09)$ & $.06(.12)$ & $.05(.11)$ & $-.27(.09)^{b}$ & $-.28(.09)^{b}$ \\
\hline & $m T B I$ & $-.44(.16)^{b}$ & $-.42(.16)^{a}$ & $-.48(.21)^{a}$ & $-.42(.20)^{a}$ & $-.42(.16)^{b}$ & $-.41(.16)^{b}$ \\
\hline & cmTBI & $-.97(.17)^{\mathcal{c}}$ & $-.94(.17)^{c}$ & $-.91(.22)^{\mathcal{c}}$ & $-.91(.22)^{\mathcal{C}}$ & $-.71(.17)^{c}$ & $-.68(.17)^{c}$ \\
\hline & msevTBI & $-1.87(.14)^{\mathcal{c}}$ & $-1.82(.14)^{\mathcal{c}}$ & $-1.98(.18)^{\mathcal{C}}$ & $-2.00(.17)^{c}$ & $-1.43(.14)^{c}$ & $-1.39(.13)^{\mathcal{c}}$ \\
\hline & $I Q$ (centered) & $.03(.01)^{c}$ & $.03(.00)^{c}$ & $.03(.01)^{b}$ & $.04(.00)^{c}$ & $.02(.01)^{a}$ & $.02(.00)^{c}$ \\
\hline & $I Q \times m T B I$ & $-.01(.01)$ & & $-.01(.02)$ & & $.01(.01)$ & \\
\hline & $I Q \times c m T B I$ & $.02(.01)$ & & $.00(.02)$ & & $.02(.01)$ & \\
\hline & $I Q \times$ msevTBI & $-.01(.01)$ & & $.01(.01)$ & & $.01(.01)$ & \\
\hline \multirow[t]{8}{*}{ Rate of Change } & Time (months) & $.03(.01)^{c}$ & $.03(.00)^{c}$ & $.022(.01)$ & $.03(.01)^{a}$ & $.01(.01)$ & $.01(.01)$ \\
\hline & Time $x$ mTBI & $.02(.01)$ & $.02(.01)$ & $.03(.02)$ & $.02(.02)$ & $.02(.01)$ & $.02(.01)$ \\
\hline & Time $x$ cmTBI & $.05(.01)^{\mathcal{c}}$ & $.05(.01)^{b}$ & $.06(.02)^{a}$ & $.06(.02)^{a}$ & $.04(.02)^{a}$ & $.04(.02)^{a}$ \\
\hline & Time $x$ msevTBI & $.11(.01)^{\mathcal{c}}$ & $.10(.01)^{\mathcal{C}}$ & $.12(.02)^{c}$ & $.12(.01)^{c}$ & $.08(.01)^{\mathcal{c}}$ & $.07(.01)^{c}$ \\
\hline & Time $x I Q$ & $-.00(.00)$ & & $.00(.00)$ & & $.00(.00)$ & \\
\hline & $\begin{array}{l}\text { Time } x I Q x \\
m T B I\end{array}$ & $.00(.00)$ & & $.00(.00)$ & & $.00(.00)$ & \\
\hline & $\begin{array}{l}\text { Time } x \text { IQ } x \\
\text { cmTBI }\end{array}$ & $.00(.00)$ & & $-.00(.00)$ & & $-.00(.00)$ & \\
\hline & $\begin{array}{l}\text { Time } x I Q x \\
\text { msevTBI }\end{array}$ & $.00(.00)$ & & $-.00(.00)$ & & $.00(.00)$ & \\
\hline Goodness of Fit ${ }^{d}$ & $A I C$ & 903.4 & 836.8 & 1282.1 & 1218.7 & 910.4 & 842.5 \\
\hline
\end{tabular}

Abbreviations: mTBI, mild TBI; cmTBI, complicated mild TBI; msevTBI, moderate to severe TBI.

Note. SAS Proc Mixed, Restricted ML. Full Level 2 conditional growth model includes the effects of TBI severity (controls as reference group), WTAR IQ (centered on population mean of 100), and their interaction on both initial status and rate of change; Final model includes only significant predictors from full model. 'Intercept' and 'Time' refer to average performance at one-month post-injury and rate of change per month, respectively, for controls with an average WTAR predicted IQ of 100 (with significance indicating that these values significantly differ from 0 ). Parameter estimates and significance testing for TBI groups are relative to controls (i.e., significance indicates that they significantly differ from those without head injury).

${ }_{\mathrm{p}}^{\mathrm{p}}<.05$;

$b_{\mathrm{p}<.01}$

$c_{\mathrm{p}}<.001$

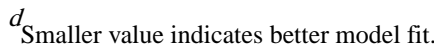

